\title{
MOLECULAR DIAGNOSIS AND TYPING OF TRYPANOSOMA CRUZI POPULATIONS AND LINEAGES IN CEREBRAL CHAGAS DISEASE IN A PATIENT WITH AIDS
}

\author{
JUAN M. BURGOS, SANDRA B. BEGHER, JORGE M. FREITAS, MARGARITA BISIO, TOMAS DUFFY, \\ JAIME ALTCHEH, RICARDO TEIJEIRO, HORACIO LOPEZ ALCOBA, FLORENCIA DECCARLINI, HECTOR FREILIJ, \\ MARIANO J. LEVIN, JORGE LEVALLE, ANDREA M. MACEDO, AND ALEJANDRO G. SCHIJMAN* \\ Laboratorio de Biología Molecular de la Enfermedad de Chagas, Instituto de Investigaciones en Ingeniería Genética y Biología \\ Molecular (INGEBI-CONICET), Buenos Aires, Argentina; Unidad 2 Clínica Médica e Infectología, Hospital Ignacio Pirovano, \\ Gobierno de la Ciudad de Buenos Aires, Buenos Aires, Argentina; Laboratorio de Parasitología y Enfermedad de Chagas, Hospital \\ de Niños Ricardo Gutiérrez, Buenos Aires, Argentina; Departamento de Bioquímica e Imunologia, ICB/UFMG, Belo Horizonte, \\ Brazil; Departamento de Fisiología y Biología Molecular y Celular, FCEyN, UBA, Buenos Aires, Argentina
}

\begin{abstract}
Trypanosoma cruzi DNA was amplified from an intracranial biopsy and peripheral blood of an HIV patient with encephalitis; this episode was indicative of AIDS and congenital Chagas disease. The analysis of a microsatellite locus revealed a multiclonal parasite population at the brain lesion with a more complex minicircle signature than that profiled in blood using restriction fragment length polymorphism (RFLP)-PCR and low stringency single primer (LSSP) PCR. Interestingly, different sublineages of T. cruzi II were detected in blood and brain by means of spliced-leader and 24s $\alpha$ ribosomal-DNA amplifications. Quantitative-competitive PCR monitored the decrease of parasitic load during treatment and secondary prophylaxis with benznidazole. The synergy between parasiticidal plus antiretroviral treatments probably allowed the patient a longer survival than usually achieved in similar episodes. This is the first case report demonstrating a differential distribution of natural parasite populations and sublineages in Chagas disease reactivation, showing the proliferation of cerebral variants not detectable in peripheral blood.
\end{abstract}

\section{INTRODUCTION}

Due to the increasing control of transmission routes mediated by vector, blood transfusion, and organ transplant, congenital transmission of Trypanosoma cruzi has emerged in terms of public health. ${ }^{1}$ Congenital cases are frequently asymptomatic, passing unnoticed unless specific tests are made. ${ }^{1,2}$ The acquired immunodeficiency syndrome (AIDS) pandemic, with 1.4 million infected people in endemic regions for Chagas disease, may lead to reactivation of quiescent chronic T. cruzi infections.,

Natural T. cruzi populations are composed of multiclonal strains with different biological properties such as replication rates, drug susceptibility, virulence, and tissue tropism, which may be implicated in the clinical forms of the disease. ${ }^{5,6}$ These strains cluster within two major phylogenetic lineages, $T$. cruzi I and T. cruzi II, with 5 lesser subdivisions within $T$. cruzi II, whose phylogenetic relationships are still under debate. $^{7,8}$ The high sensitivity of PCR-based typing strategies provides direct assessment of parasite genetic diversity in clinical specimens without the need of culture isolation, ${ }^{9-11}$ which may underestimate natural population complexity due to strain selection during culture expansion. Herein, we report the differential diagnosis of chagasic encephalitis in a patient with presumptive cerebral toxoplasmosis due to AIDS, applying PCR in a surgical sample of brain tissue and peripheral blood specimens, not detectable using current parasitological methods. Furthermore, PCR-based identification of $T$. cruzi lineages and profiling of microsatellite and minicircle signatures were assessed directly from the clinical specimens, providing evidence of a differential host tissue distribution of natural parasitic populations.

\footnotetext{
* Address correspondence to Alejandro G. Schijman, Ph.D., Laboratorio de Biología Molecular de la Enfermedad de Chagas, Instituto de Investigaciones en Ingeniería Genética Biología Molecular (INGEBI-CONICET), Buenos Aires, Argentina. E-mail: schijman@ dna.uba.ar
}

\section{CASE REPORT}

A 29-year-old HIV-positive Argentinian man, born in Buenos Aires, was hospitalized in February 2003 with left hemiparesis and a history of protracted headaches of 1 month of evolution. Magnetic resonance imaging showed multiple masses on the left brain hemisphere (Figure 1A) and antitoxoplasma IgG serodiagnosis was positive, although near the cutoff line $(6 \mathrm{IU} / \mathrm{mL})$. Consequently, treatment of presumptive cerebral toxoplasmosis was implemented. As the patient did not respond to the specific treatment, an intracranial biopsy guided by CT-scan was indicated to determine the etiology of the encephalitis. Histologic analysis exhibited edema and high lymphocyte counts but did not reveal any protozoan forms. Thus, we applied PCR procedures targeted to T. gondii $\mathrm{B}^{12}$ and $T$. cruzi minicircle $(\mathrm{kDNA})^{13}$ and satellite (satDNA) sequences ${ }^{14}$ from DNA extracts of the brain tissue specimen, which only revealed $T$. cruzi DNA, indicative of cerebral Chagas disease. Microhematocrit analysis ${ }^{2}$ did not show any bloodstream forms, but the high sensitivity of kDNA-PCR $(0.4 \log \text { parasite genome equivalents } / \mathrm{mL})^{13}$ allowed detection of $T$. cruzi DNA in peripheral blood. Besides, anti-T. cruzi serodiagnosis also resulted positive (ELISA titer R: 2.7, cutoff $<1$, and indirect hemaglutination titer $1 / 256$, cutoff $<1: 32$ ). Thus, on the basis of $T$. cruzi DNA amplification and seroreactivity, the patient received a final diagnosis of chagasic encephalitis. Accordingly, trypanocidal therapy with benznidazole $\left(5 \mathrm{mg} \mathrm{kg}^{-1}\right.$ day $\left.^{-1}\right)$ was administered. Treatment (tmt) response was monitored by competitive-quantitative PCR (Q-PCR) on peripheral blood, ${ }^{13}$ which initially revealed approximately 280 parasites genome equivalents/mL of blood (2.4 log. pge/mL) (Figure 1B; April 2003). Treatment regimen was modified 25 days later because the patient presented polymorphic erythema. At that time, the parasitic load had decreased to $1.4 \mathrm{log} \cdot \mathrm{pge} / \mathrm{mL}$ (Figure 1B; May 2003). After the remission of symptoms, tmt was restarted for 60 days leading to further decrease of parasitemia to undetectable loads (Q-PCR below $1.09 \mathrm{log}$. and kDNAPCR below 0.4 log. pge/mL; Figure 1B, August 2003). There- 

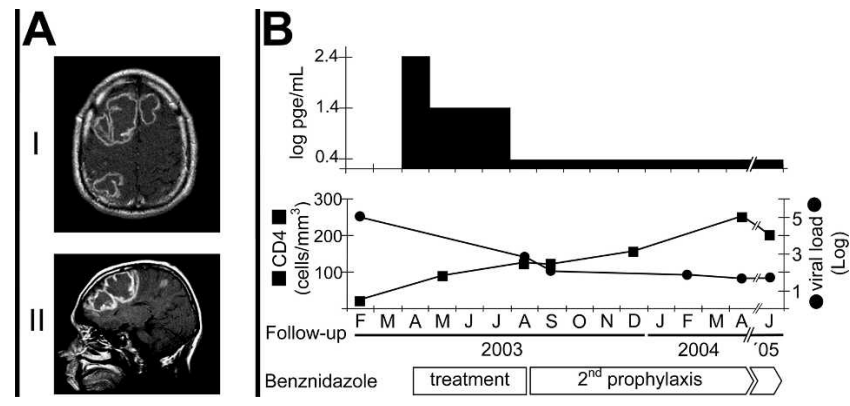

Figure 1. A, Magnetic resonance imaging (T1 with gadolinium) showing cerebral mass lesions at time of diagnosis of encephalitis (February 2003). I, transversal section; II, sagital section. B, Diagnosis and follow-up of the AIDS patient with chagasic encephalitis. The parasitic load was estimated by means of quantitative-competitive PCR. Log. pge $/ \mathrm{mL}$ : logarithm of parasite genomic equivalents $/ \mathrm{ml}$ of blood. $\square$ : CD4 cells count. ๑: HIV load.

after, secondary prophylaxis with benznidazole was initiated (5 mg kg-1 day $^{-1}$ twice a week) with persistently negative PCR findings (Figure 1B; August 2003 to February 2005) accompanied by a good clinical evolution without neurologic symptoms. Magnetic resonance imaging showed a reduction of perilesional edema although brain mass did not remit (not shown); two cerebral spinal fluid samples withdrawn during post-treatment follow-up were negative when examined by microscopy and kDNA-PCR (not shown). The anti-T. cruzi immunologic response persisted positive throughout followup. Antiretroviral tmt (ART) promoted a decrease of HIV viral load from $>500,000$ copies $/ \mathrm{mL}$ to undetectable levels (< $1.7 \log \cdot$ HIV copies $/ \mathrm{mL}$ ), accompanied with an increase of CD4+ cell counts from $22 / \mathrm{mm}^{3}$ to $194 / \mathrm{mm}^{3}$ (Figure 1B). After 30 months of survival, the patient continues under anti- $T$. cruzi prophylaxis, ART, and anticonvulsive tmt. This study was approved by the hospital review board and ethical committees of the institutions; written informed consent was required in agreement with the guidelines of the International Conference on Harmonization.

Molecular profiling of parasitic populations and lineages involved in Chagas disease reactivation. To characterize the genetic diversity of the patients parasitic populations found in peripheral blood and brain tissue samples, the 330-bp kDNA amplicons were profiled using low stringency single primer (LSSP) and restriction fragment length polymorphism (RFLP) PCR strategies ${ }^{10,11}$ (Figure 2). Interestingly, minicircle signatures from brain tissue parasites exhibited a different profile than that obtained from bloodstream parasites at time of diagnosis (Figure 2A). The minicircle signatures detected in brain tissue showed a higher number of amplicons than those in blood, suggesting a higher diversity of parasite variants. This diversity was further explored by amplification of a microsatellite marker, known as TAC $15,{ }^{9}$ which revealed a multiclonal population in the brain chagoma (amplicons of 128, 131, and $134 \mathrm{bp}$; Figure 2B, br) whereas a homozygous and conserved population was detected in bloodstream at time of diagnosis and during treatment follow-up (amplicons of $131 \mathrm{bp}$; Figure $2 \mathrm{~B}, \mathrm{bl}_{0}$ and $\mathrm{bl}_{45}$, respectively).

The bloodstream parasite minicircle signatures were characterised during antiparasite treatment (Figures 2A and 2C). The RFLP-PCR and LSSP-PCR profiles obtained from $T$. cruzi $\mathrm{kDNA}$ in blood collected 45 days after initiation of tmt

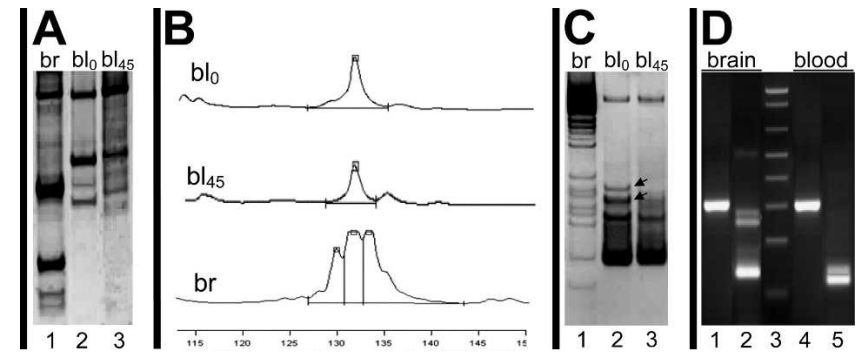

FIGURE 2. Molecular typing of T. cruzi lineages and populations implicated in cerebral Chagas reactivation. A, LSSP-PCR profiles of 330-bp kDNA amplicons. B, Electrofluorogram of T. cruzi "TAC 15" microsatellite locus (values in the scale given in bp). C, AluI + HinfI RFLP-PCR of 330-bp kDNA amplicons. LSSP-PCR and RFLP-PCR were analyzed by $10 \%$ acrylamide gel electrophoresis and Sybr Green dye staining. $\mathrm{Br}$, brain tissue; $\mathrm{bl}_{0}$, blood sample collected before tmt with benznidazole; $\mathrm{bl}_{45}$, blood sample collected 45 days after start of tmt. D, Ethidium bromide-stained 3\% agarose gel showing PCR-based typing of T. cruzi lineages. Lanes 1 and 4, spliced-leaderbased PCR; Lanes 2 and 5, 24s $\alpha$ ribosomal DNA-based heminested PCR using primers D75-D76 (first round) and D71-D76 (second round $)^{15}$; Lane 3, 100-bp molecular weight marker.

revealed a reduction in the number and/or the relative presence of certain minicircle subclasses compared with the patterns of the pretreatment population (Figures $2 \mathrm{~A}$ and $2 \mathrm{C}$, lanes $\mathrm{bl}_{0}$ and $\left.\mathrm{bl}_{45}\right)$. These findings suggested a differential tmt response of certain parasite variants until the complete clearance of the parasitemia at the end of tmt.

To identify the parasite lineages in peripheral blood and brain chagoma, the intergenic region of the spliced-leader genes was amplified by a multiplex PCR assay, ${ }^{15}$ revealing $T$. cruzi II in both clinical specimens (Figure 2D, lanes 1 and 4). Furthermore, heminested amplification of the D7 domain of the $24 \mathrm{~s} \alpha$ ribosomal RNA genes ${ }^{15}$ allowed to distinguish $T$. cruzi II b/e (rDNA group 2/2) at the brain lesion and T. cruzi IId (rDNA group 1/2) in bloodstream (Figure 2D, lanes 2 and 5 , respectively).

\section{DISCUSSION}

According to Centers for Diseases Control and Prevention, 3 of the 12 case-defining opportunistic infections for AIDS Group IV are parasitoses, namely toxoplasmosis, cryptosporidiosis, and isosporidiosis. However, in endemic regions for Chagas disease, T. cruzi should be included among the potential opportunistic pathogens indicative of AIDS. ${ }^{3,4}$ In this scenario, the PCR provided a rapid differential and sensitive diagnosis of $T$. cruzi reactivation allowing prompt administration of specific chemotherapy, which contributed to the patients long survival. This case further illustrated the usefulness of Q-PCR to follow-up parasitic response to treatment and secondary prophylaxis. Moreover, prospective Q-PCR could aid in assessing the risk of reactivation to implement primary prophylaxis. It is worth noting that the synergy of parasiticidal secondary prophylaxis plus antiretroviral treatment may have allowed the patient a longer survival than usually achieved in similar episodes.,

To date, few cases of chagasic reactivation due to AIDS have been described in detail., ${ }^{3,4}$ The typical epidemiologic sequence of events was to acquire $T$. cruzi infection in rural areas of endemicity and to move to urban centers where HIV 
infection was acquired, leading to opportunistic disease as immunosuppression ensued. ${ }^{3,4}$ However, the case reported herein most likely acquired Chagas disease through vertical transmission. This was assumed because a) the patient was born in a nonendemic area, b) did not have any contact with triatomine vectors, did not receive transfusions, did not use intravenous drugs, and c) his mother was seropositive for Chagas disease. Congenital Chagas disease cannot be prevented, but early diagnosis and treatment achieve very high cure rates. $^{2,13}$ This stresses out the public health importance of surveying all newborns to seropositive mothers in nonendemic areas for Chagas disease.

The role of $T$. cruzi parasitemia and genetic make-up on the onset of chagasic reactivation due to AIDS, as well as the impact of HIV infection in T. cruzi genetic diversity, are poorly explored fields of clinical parasitology. ${ }^{16,17}$ Comparative zymodeme studies of parasite stocks obtained from HIVpositive patients with $T$. cruzi coinfection did not reveal significant differences regarding the tissue tropism or repartition of the strains. ${ }^{16}$ Remarkably, this case is the first evidence of a differential distribution of $T$. cruzi populations associated with reactivation, showing the proliferation of cerebral parasite multiclonal populations, belonging to a sublineage not detectable in peripheral blood, indicative of lineagehistotropism in Chagas disease.

Received June 9, 2005. Accepted for publication July 8, 2005.

Acknowledgments: We are indebted to the work of Dr. Gustavo Rolando and Alejandro Seoane. This work was supported by grants from the World Health Organization-Tropical Diseases Research projects (ID 20285 and 990893) and Bunge \& Born Private Foundation of Argentina (to A.G.S.), CONICET (PEI 2000), University of Buenos Aires, FONCyT BID 802/OC-AR PICT 01421, PICT 02030, and PRONEX FAPEMIG/CNPQ. The work of Dr. M.J. Levin is partially supported by an International Research Scholar grant from the Howard Hughes Medical Institute (Chevy Chase, MD). M.J.L. and A.G.S. are members of the National Research Council (CONICET) Research Career Program. J.A. and H.F. are members of the research career of Buenos Aires city Government.

Authors' addresses: Juan M. Burgos, Laboratorio de Biología Molecular de la Enfermedad de Chagas, Instituto de Investigaciones en Ingeniería Genética y Biología Molecular (INGEBI-CONICET), Buenos Aires, Argentina and Departamento de Fisiología y Biología Molecular y Celular, FCEyN, UBA, Buenos Aires, Argentina. Margarita Bisio, Tomas Duffy, Mariano J. Levin, and Alejandro G. Schijman, Laboratorio de Biología Molecular de la Enfermedad de Chagas, Instituto de Invesigaciones en Ingeniería Genética y Biología Molecular (INGEBI-CONICET), Buenos Aires, Argentina. Sandra B. Begher, Ricardo Teijeiro, Horacio Lopez Alcoba, Florencia Deccarlini, and Jorge LeValle. Unidad 2 Clínica Médica e Infectología, Hospital Ignacio Pirovano, Gobierno de la Ciudad de Buenos Aires, Buenos, Aires, Argentina. Jorge M. Freitas and Andrea M. Macedo, Departamento de Bioquímica e Imunología, ICB/UFMG, Belo Horizonte, Brazil. Jaime Altcheh and Hector Freilij, Laboratorio de Parasitología y Enfermedad de Chagas, Hospital de Niños Ricardo Gutiérrez, Buenos Aires, Argentina.

Reprint requests: Alejandro G. Schijman, Ph.D., Vuelta de Obligado 2490, 2nd floor, Buenos Aires 1428, Argentina, Telephone: 054114783-2871, Fax: 05411-4786-8578, E-mail: schijman@dna.uba.ar.

\section{REFERENCES}

1. Gurtler RE, Segura EL, Cohen JE, 2003. Congenital transmission of Trypanosoma cruzi infection in Argentina. Emerg Infect Dis 9: 29-32.

2. Freilij H, Altcheh J, 1995. Congenital Chagas' disease. Diagnostic and clinical aspects. Clin Inf Dis 21: 551-555.

3. Corti M, 2000. AIDS and Chagas disease. AIDS Patient Care STDs 14: $581-588$.

4. Cahn P, Badaró R, Freilij H, 2001. Other parasitic infections. Crowe S, Hoy J, Mills J, eds. Management of the HIV-Infected Patient. 2nd ed. Taylor \& Francis Group, London, U.K., 31: 513-525.

5. Macedo AM, Pena SDJ, 1998. Genetic variability of Trypanosoma cruzi: implications for the pathogenesis of Chagas disease. Parasitol Today 14: 119-123.

6. Macedo AM, Machado CR, Oliveira R, Pena SDJ, 2004. Trypanosoma cruzi: genetic structure of populations and relevance of genetic variability to the pathogenesis of Chagas disease. Mem Inst Oswaldo Cruz 99: 1-12.

7. Anonymous, 1999. Recommendations from a satellite meeting. Mem Inst Oswaldo Cruz 94: 429-432.

8. Brisse S, Barnabe C, Tibayrenc M, 2000. Identification of six Trypanosoma cruzi phylogenetic lineages by random amplified polymorphic DNA and multilocus enzyme electrophoresis. Int J Parasitol 30: 35-44.

9. Macedo AM, Pimenta JR, Aguiar RS, Melo AIR, Chiari E, Zingales B, Pena SDJ, Oliveira RP, 2001. Usefulness of microsatellite typing in population genetic studies of T. cruzi. Mem Inst Oswaldo Cruz 96: 407-413.

10. Vago AR, Macedo AM, Oliveira RP, Andrade LO, Chiari E, Galvao LM, Reis D, Pereira ME, Simpson AJ, Tostes S, Pena SD, 1996. Kinetoplast DNA signatures of Trypanosoma cruzi strains obtained directly from infected tissues. Am J Pathol 149: 2153-2159.

11. Solari A, Munoz S, Venegas J, Wallace A, Aguilera X, Apt W, Breniere SF, Tibayrenc M, 1992. Characterization of Chilean, Bolivian, and Argentinian Trypanosoma cruzi populations by restriction endonuclease and isoenzyme analysis. Exp Parasitol 75: 187-195.

12. Ho-Yen DO, Joss AW, Balfour AH, Smyth ET, Baird D, Chatterton JM, 1992. Use of the polymerase chain reaction to detect Toxoplasma gondii in human blood samples. J Clin Pathol 45: 910-913.

13. Schijman AG, Altcheh J, Burgos JM, Biancardi M, Bisio M, Levin MJ, Freilij H, 2003. Aetiological treatment of congenital Chagas' disease diagnosed and monitored by the polymerase chain reaction. J Antimicrob Chemother 52: 441-449.

14. Schijman AG, Vigliano C, Burgos J, Favaloro R, Perrone S, Laguens R, Levin MJ, 2000. Early diagnosis of recurrence of Trypanosoma cruzi infection by polymerase chain reaction after heart transplantation of a chronic Chagas' heart disease patient. J Heart Lung Transplant 19: 1114-1117.

15. Souto RP, Fernandes O, Macedo AM, Campbell DA, Zingales B, 1996. DNA markers define two major phylogenetic lineages of Trypanosoma cruzi. Mol Biochem Parasitol 83: 141-152.

16. Perez-Ramirez L, Barnabe C, Sartori AM, Ferreira MS, Tolezano JE, Nunes EV, Burgarelli MK, Silva AC, ShikanaiYasuda MA, Lima JN, Da-Cruz AM, Oliveira OC, Guilherme C, Bastrenta B, Tibayrenc M, 1999. Clinical analysis and parasite genetic diversity in human immunodeficiency virus/ Chagas' disease coinfections in Brazil. Am J Trop Med Hyg 61: 198-206.

17. Sartori AM, Caiaffa-Filho HH, Bezerra RC, Do S, Guilherme C, Lopes MH, Shikanai-Yasuda MA, 2002. Exacerbation of HIV viral load simultaneous with asymptomatic reactivation of chronic Chagas' disease. Am J Trop Med Hyg 67: 521-523. 